\title{
OPTICAL PROPERTIES AND RADIATION RESPONSE OF Li ION-DOPED CSI SCINTILLATOR CRYSTAL
}

\author{
Maria da Conceição Costa Pereira' ${ }^{*}$, Tufic Madi Filho, \\ José Roberto Berretta', Lucas Faustino Tomaz', Miriam Nieri Madi'
}

\author{
1Instituto de Pesquisas Energéticas e Nucleares (IPEN / CNEN - SP), São Paulo, SP, Brazil \\ ${ }^{2}$ Universidade Paulista - UNIP-SP, Software Engineering Research Group, São Paulo, Brazil
}

\begin{abstract}
Scintillators are materials that convert the energy of ionizing radiation into a flash of light. Due to the existence of different types of scintillators, they are classified into three groups according to their physicochemical characteristics, namely, inorganic, organic and gaseous scintillators. Among the inorganic crystals, the most frequently used as scintillator consist of alkali metals, in particular alkaline iodides. Scintillation materials have many applications, for instance in medical imaging, security, physics, biology, non-destructive inspection and medicine. In this study, lithium doped CsI scintillator crystals were grown using the vertical Bridgman technique. The concentration of the lithium doping element ( $\mathrm{Li}$ ) studied was $1 \mathrm{O}^{-4} \mathrm{M}$ to $1 \mathrm{O}^{-1} \mathrm{M}$. Analyses were carried out to evaluate the developed scintillators with regard to luminescence emission and optical transmittance. The luminescence emission spectra of these crystals were measured with a monochromator for gamma radiation from ${ }^{137 C s}$ source excitation. The determination of the dopant distribution along the crystalline axis allowed the identification of the region with Li concentration uniformity, which is the region of the crystalline volume indicated for use as a radiation detector. The crystals were excited with neutron radiation from AmBe source, with the energy range of $1 \mathrm{MeV}$ to 12 $\mathrm{MeV}$. As neutron sources also generate gamma radiation, which can interfere with the measurement, it is necessary that the detector be able to discriminate the presence of such radiation. Accordingly, experiments were performed using gamma radiation in the energy range of $59 \mathrm{keV}$ to $1333 \mathrm{keV}$ in order to verify the ability of the detector to discriminate the presence of different types of radiation.
\end{abstract}

Keywords: Scintillator, radiation detector, crystal growth, neutron source

\section{INTRODUCTION}

The use of scintillation to detect radiation is a century old. A history of the discovery of important inorganic scintillator materials is considered in the sense that they either became commercially available and widely used or triggered further developments or new research areas $[1,2]$.

Among the types of detectors, scintillators meet the diverse needs in the field of radiation detection. Scintillators are materials capable of producing light when ionizing radiation dissipates its energy in their midst. Due to the existence of different types of scintillators, they are classified into three groups depending on their physicochemical characteristics, namely inorganic scintillators, organic scintillators and gaseous scintillators. Among the inorganic crystals, the most frequently used as scintillators consist of alkali metals, in particular alkali halide [3,4]. The inorganic crystals have been studied for use as radiation sensors, since the 50s [5 ]. Since then, inorganic crystals have been studied in various fields of science and engineering, such as high energy physics, nuclear medicine, geophysics, environmental monitoring, with regard to devices and systems for security inspection, non-destructive testing, space physics, astronomy, etc. A better understanding of the various mechanisms of scintillation has enabled the use of new materials for detecting various types of radiation [6]. Although many of the foundations of physics, essential to the process of scintillation, have been studied, the need to improve these materials has been important in the research and development of materials scintillators.

Scintillators containing lithium (Li), already possessing natural abundance of $7.5 \%$ in ${ }^{6} \mathrm{Li}$, are attractive because interaction with thermal neutrons produces an alpha particle and triton totaling 4.78 $\mathrm{MeV}$ which can be readily absorbed and converted to scintillation light. For neutron detection, a constituent ion with a high neutron absorption section such as ${ }^{6} \mathrm{Li}$ is necessary. Another attractive feature of Li is its very low cross section for gamma interactions [7]. The neutron active material initiates the light production by releasing energy charged particles or gamma rays when the neutron is captured. After the initial interaction with the neutron occurs, the detection process is the same as if the light were produced by a gamma ray. Because the scintillator is also a gamma ray detector,

*macoper@ipen.br 
its gamma ray sensitivity is generally very high. There are, however, several possible configurations with good neutron detection efficiency and low gamma ray sensitivity.[8]

In this paper, optical properties and radiation response of the $\mathrm{Li}$ ions doped cesium iodide (CsI:Li) crystals, were studied.

\section{METHODS}

The vertical Bridgman technique $[9,10]$ was used to grow CsI:Li crystals. The concentration of the lithium doping was in the range of $10^{-4} \mathrm{M}$ to $10^{-1} \mathrm{M}$. Pure CsI (99.99\%) and LiI (99.99\%) according to the stoichiometric ratio were loaded in the quartz crucibles and then heated in a vacuum of $10^{-4} \mathrm{~Pa}$ to eliminate the residual humidity. The crucibles were maintained at a temperature of $50{ }^{\circ} \mathrm{C}$ above the melting point of cesium iodide for $10 \mathrm{~h}$ to ensure homogeneity of the melt. Only then, the displacement of the crucible started towards an optimal temperature gradient of the furnace at a speed of $1 \mathrm{~mm} \mathrm{~h}^{-1}$ by means of a displacement system with programmable logic control.

Luminescence emission spectra for the CsI:Li and pure CsI crystals were evaluated via the photometric analysis of the stimulated crystals, with a radioactive source of ${ }^{137} \mathrm{Cs}(662 \mathrm{keV})$ positioned in front of each sample coupled to the monochromator input. The pulses of light from the scintillators were converted into electrical impulses by means of a photomultiplier tube, optically coupled to the output of the monochromator. The associated electronics for analysis consisted primarily of the conventional ORTEC nuclear instrumentation electronics.

Due to the phenomenon of the segregation of impurities in the crystal growth process, it was necessary to investigate the behavior of the lithium doping element in its distribution along the crystalline volume. This investigation was carried out using the argon plasma optical emission spectrometry (ICP OES) analytical technique, which is based on the observations of radiative emissions of the constituent elements of the sample in a plasma, usually argon. In a plasma, free atoms and ions are obtained which can be detected and quantitatively determined as a function of emission intensities at specific wavelengths. Because it is a destructive analysis, only $\mathrm{CsI}: \mathrm{Li}^{10^{-3}} \mathrm{M}$ crystal was used since it has a good response to neutron and gamma radiation. This crystal was cut into 10 slices of $14 \mathrm{~mm}$ thickness each. From each slice a $100 \mathrm{mg}$ sample was taken from the upper surface and solubilized with hydrochloric acid $(\mathrm{HCl}) 1 \mathrm{M}$, then made up to the $25 \mathrm{~mL}$ volumetric flask meniscus. Therefore, 10 samples were prepared for analysis.

In the study of gamma radiation and neutron radiation response, the crystals were machined, polished and coupled directly to the bi-alkaline photomultiplier (model RCA 8575, 21 pins) using a viscosity of 0.5 McStokes silicone grease (Dow Corning) as an optical interface. The electronic modules used for the treatment of the signals from the photomultiplier tube consisted of conventional ORTEC electronics. The crystals were prepared to be $26 \mathrm{~mm}$ in diameter and $14 \mathrm{~mm}$ thick for both experiments.

Gamma radiation sources were employed, with energies ranging from $59 \mathrm{keV}$ to $1333 \mathrm{keV}$. The radioactive sources were positioned in the center of the upper face of the crystal. The photomultiplier operating voltage was $2400 \mathrm{~V}$ for the detection of gamma rays. The accumulation time in the counting process was $600 \mathrm{~s}$.

The neutron detection efficiency of the crystals was measured in two different positions, in the first (position I) the AmBe source with the energy of $1 \mathrm{MeV}$ to $12 \mathrm{MeV}$ was positioned at a distance of $70 \mathrm{~mm}$ from the photomultiplier tube. The photomultiplier operating voltage was $2400 \mathrm{~V}$. The accumulation time in the counting process was $600 \mathrm{~s}$. The fluency was $2.6 \times 10^{6}$ neutrons / second.

In the second (position II), the AmBe source was positioned at a distance of $70 \mathrm{~mm}$ from the photomultiplier tube, using $70 \mathrm{~mm}$ paraffin block as the interface. A foil of $\mathrm{Cd}$ was placed around the crystal and the photomultiplier tube. The foil of Cd was used to avoid the scattered neutron contribution, as shown in Fig. 1. The photomultiplier operating voltage was $1900 \mathrm{~V}$. The accumulation time in the counting process was $1800 \mathrm{~s}$. The fluency was $2.6 \times 10^{6}$ neutrons / second.

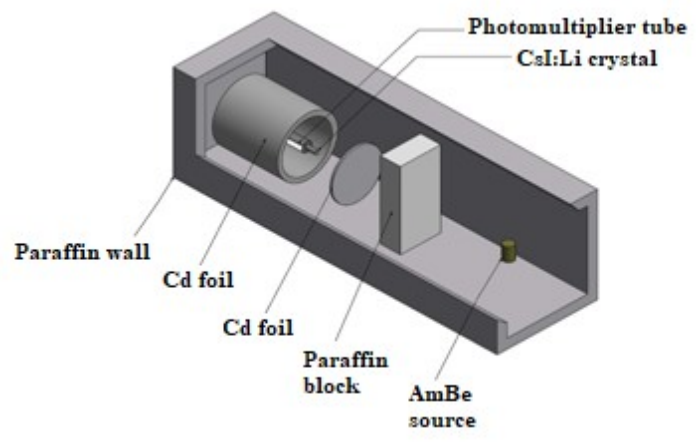

Figure 1. Exploded schematic representation of the array used for measurements, (position II).

\section{RESUlts AND DisCUSSION}

In this paper, the scintillator crystals were obtained in lithium doped CsI matrix, with a molar concentration of $10^{-4}, 10^{-3}, 10^{-2}, 10^{-1}$ and pure CsI. The crystals were grown to $27 \mathrm{~mm}$ in diameter and $140 \mathrm{~mm}$ in length. Fig. 2 shows a CsI:Li crystal: (a) crystal in the geometric shape of the crucible, (b) cut crystal $14 \mathrm{~mm}$ thick.

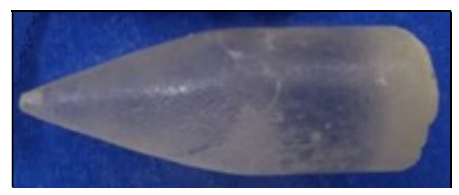

(a)

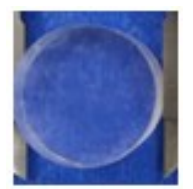

(b)
Figure 2. Scintillator crystals CsI: $\mathrm{Li}_{10}{ }^{-3} \mathrm{M}$ (a) crystal in the geometric shape of the crucible, (b) cut crystal $14 \mathrm{~mm}$ thick.

The luminescence spectra in the function of the wavelength for the pure CsI and CsI:Li $10^{-4} \mathrm{M}, 10^{-3} \mathrm{M}$, $10^{-2} \mathrm{M}, 10^{-1} \mathrm{M}$ crystals excited with gamma radiation

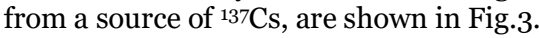




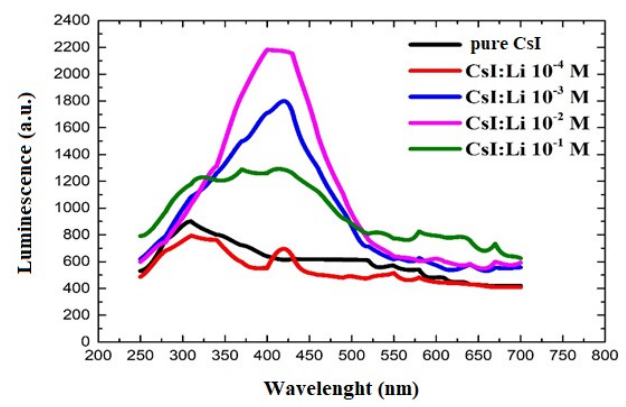

Figure 3. Luminescence spectra for pure CsI and CsI: ${\mathrm{Li} 10^{-4}}^{-4}$ $\mathrm{M}, 10^{-3} \mathrm{M}, 10^{-2} \mathrm{M}, 10^{-1} \mathrm{M}$ crystals using gamma radiation of the ${ }^{137} \mathrm{Cs}$ source

As can be seen in Fig. 3, the maximum luminescence emission wavelength is characteristic for each crystal. The maximum intensity of luminescence at the wavelength of $320 \mathrm{~nm}$, found for pure CsI crystal, is in agreement with the literature [11]. The origin of this luminescence in the crystal is attributed to the characteristic of the material in its pure form. As shown in Fig. 3, the luminescence spectra for the CsI:Li crystals showed light emission peaks at wavelength close to $420 \mathrm{~nm}$ for all concentrations of the dopant element. In addition to an intense peak at $420 \mathrm{~nm}$, the emission peak with a lower intensity near $320 \mathrm{~nm}$ was also observed in the luminescence spectrum of the CsI:Li crystals.

The emission at $420 \mathrm{~nm}$ can be attributed to the presence of lithium ions, while the origin of the peak of a lower intensity at the wavelength around $320 \mathrm{~nm}$ could be attributed to the intrinsic luminescence from the pure CsI crystal. As may be observed in Fig. 3, the CsI:Li $10^{-2} \mathrm{M}$ crystal showed the highest luminescence intensity in the range studied. The CsI:Li $10^{-1} \mathrm{M}$ crystal showed a significant decrease in luminous intensity when compared to the other concentrations. This decrease in luminescence can be attributed to the saturation of the crystal lattice.

For scintillators intended for the construction of radiation detectors, a homogeneous distribution of the dopants over a wide region of the crystalline volume is desirable, since the luminescence emission efficiency depends on this homogeneity [12].

In the study of lithium dopant distribution along the crystalline volume, it was added to the CsI matrix with $10^{-3}$ molar fraction. In the course of the crystallization process this concentration is modified resulting in a concentration gradient, which is an important quality control parameter in obtaining scintillating crystals. The results of the lithium concentration in 10 regions of the CsI: $\mathrm{Li}_{10}^{-3} \mathrm{M}$ crystalline block obtained by (ICP OES) are shown in Fig. 4.

The results obtained from the argon plasma optical emission spectrometric analysis indicated a higher concentration at the top of the crystal (slice 10), with a subsequent decrease in the early growth phase. A considerable uniformity in lithium concentration was found between slice 2 and slice 7 , as can be seen in Fig. 4, which is the region of the crystalline block suitable for use as a radiation detector. From slice 9, the concentration of lithium grows abruptly and consequently this region must be neglected. The initial growth region, slice 1, had slightly higher lithium concentration and was also discarded. The increase in lithium concentration in the final growth region was already expected, as impurities tend to migrate to the final phase of growth due to the segregation phenomenon.

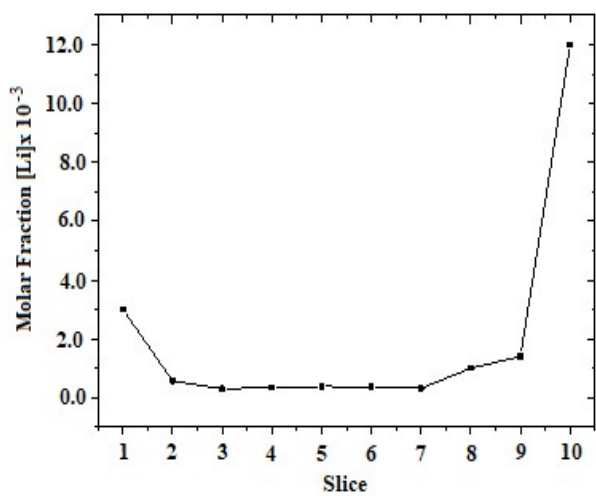

Figure 4. Distribution of

lithium concentration in crystalline volume

Considering that the crystalline volume needs to present homogeneity in the dopant concentration, the obtained results showed that $70 \%$ of the crystal is suitable for use as a radiation detector.

The investigation of the crystals as to their performance as a gamma radiation detector was carried out using emitting sources with energies in the range of $59 \mathrm{keV}$ to $1333 \mathrm{keV}$. The radiation sources were placed in contact with the crystals. Individually obtained spectra in response to the gamma excitation undergone by crystals are obtained for the four concentrations and for the pure CsI crystal. Fig. 5 and Fig. 6 show the spectra obtained in the detection of gamma radiation.

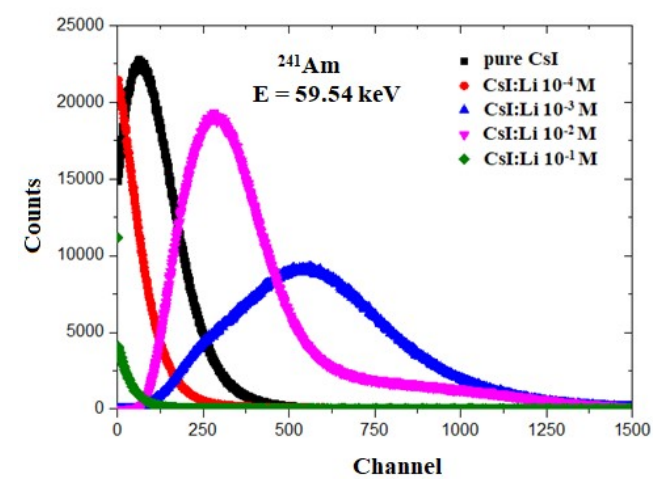

Figure 5. Detection spectra obtained with CsI:Li and pure CsI crystals excited with gamma radiation from a ${ }^{241}$ Am source

The addition of lithium dopant in CsI crystals significantly influenced its behavior as a gamma detector. In Fig. 5 and 6 it can be seen that the pulse height is dependent on Li concentration.

Considering that the spectra were obtained in the same arrangement, under the same measurement conditions and with the same electronic adjustment, it can be noted that, as the energy of the gamma emitting 
source increases, the spectra change to corresponding channels at higher energies. Therefore, detection with these crystals has the characteristic of proportional detector. In the spectra obtained with CsI:Li crystals excited with gamma radiation with this electronic arrangement it was possible to verify a good distinction in energy by arranging it in different channels, such as the ${ }^{241} \mathrm{Am}(59 \mathrm{keV})$ power source in the first 1500 channels and positioning the ${ }^{60} \mathrm{Co}(1173 \mathrm{keV}, 1333 \mathrm{keV})$ source between channels 3000 and 4000 . In Fig.5 only 1500 channels have been presented, since the power source is low.

The study of neutron radiation excited crystals performance was performed with an AmBe source with the energy of $1 \mathrm{MeV}$ to $12 \mathrm{MeV}$. The spectra were obtained individually with the neutron emitting source positioned $70 \mathrm{~mm}$ from the crystals. The Fig. 7 show the spectra obtained in the detection of neutron radiation.

The CsI:Li crystals $10^{-2} \mathrm{M}$ and $10^{-3} \mathrm{M}$ showed detection spectra with a higher volume of counts than the other crystals, as opposed to comparisons with gamma radioactive sources. The significant detection improvement could be attributed to the ability of the lithium doping element to interact with neutrons, justified by its high absorption shock section (640 barns). This demonstrates that lithium doped CsI crystals can be exploited in mixed-field neutron detection arrangements as they will not suffer significant gamma radiation interference and can provide more reliable neutron detection [13].

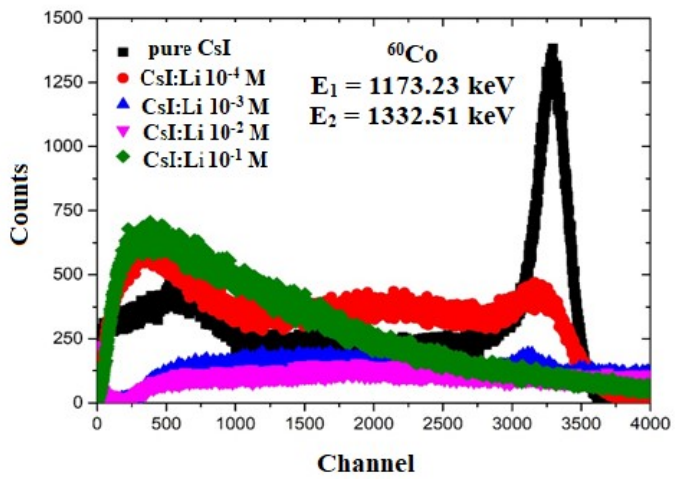

Figure 6. Detection spectra obtained with CsI:Li and pure CsI crystals excited with gamma radiation from a ${ }^{6 \circ} \mathrm{Co}$ source

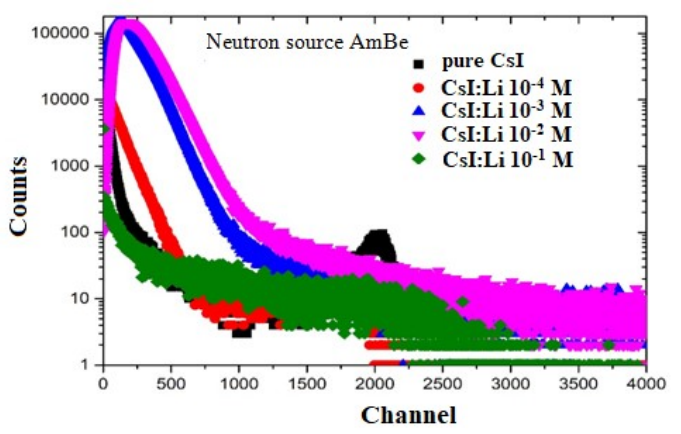

Figure 7. Detection spectra obtained with CsI:Li and pure CsI crystals excited with neutron radiation from a AmBe source

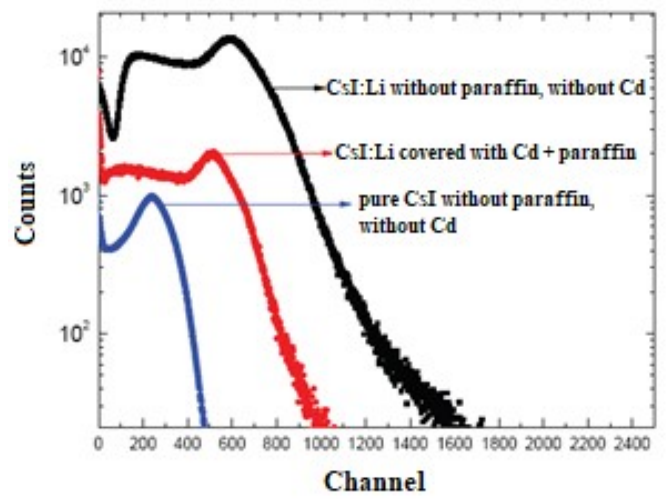

Figure 8. Results of the measurements using pure CsI and CsI:Li crystals and neutron source, with paraffin and Cd foil

For neutrons to become thermalized it is necessary that they undergo collisions when crossing the field, losing part of their energy until they reach thermal range. For this to occur, moderator materials are used, which have the function of reducing the velocity. Hydrogenated materials, e.g. paraffin and polyethylene, are rich in hydrogen and are considered good moderators.

The curve shown in Fig. 8 illustrates the results of the neutron radiation from an AmBe source using the CsI:Li crystal and pure CsI. Paraffin was used for the thermalization of fast neutrons (position II). The largest number of counts obtained with the crystals CsI:Li, when excited with radiation from a neutron AmBe source, compared to the number of counts obtained with pure CsI crystal, demonstrates the incorporation of lithium in the crystal structure. The crystals doped with lithium showed a good efficiency since neutrons have a high cross-section for the reaction $(n, \alpha)$. Even though the crystal is small, the products of this reaction $\mathrm{n}+{ }^{6} \mathrm{Li}(7.5 \%) \rightarrow 3 \mathrm{H}(2.75$ $\mathrm{MeV})+\alpha$, were detected this crystalline volume.

\section{CONCLUSION}

The vertical Bridgman technique used in this study was suitable for the growth of lithium doped CsI crystals.

The luminescence maximum emission peak spectra at $420 \mathrm{~nm}$ showed a good overlap with the quantum efficiency spectrum of the alkaline photomultiplier tubes, demonstrating the feasibility of using CsI:Li crystals as radiation detectors.

The analysis of the dopant concentration in ten (10) slices with molar fraction $10^{-3}$ demonstrated that $70 \%$ of the crystalline volume showed lithium dopant concentration homogeneity. The distribution of lithium in the crystalline volume showed an adequate behavior, with a higher concentration at the beginning of the growth process, remaining practically uniform between slices 2 and 7, and with a significant increase in dopant at the end of growth.

The addition of the Li to the CsI matrix resulted in crystals with promising results, when excited with gamma and neutron radiation. 
M. C. Costa Pereira et al., Optical properties and radiation response..., RAP Conf. Proc., vol. 4, 2019, 131-135

\section{REFERENCES}

1. C. W. E. Eijk, "Inorganic-scintillator development," Nucl. Inst. Methods Phy. Res., vol. 460, no. 1, pp. 1 - 14, Mar. 2001. DOI: 10.1016/So168-9002(00)01088-3

2. T. Yanagida, "Inorganic scintillating materials and scintillation detectors," Proc. Jpn. Acad. Ser. B Phys. Biol. Sci., vol. 94, no. 2, pp. 75 - 97, Feb. 2018.

DOI: $10.2183 /$ pjab.94.007

PMid: 29434081 PMCid: PMC 5843761

3. G. F. Knoll, Radiation Detection and Measurement, 4th ed., New York (NY), USA: J. Wiley \& Sons, 2010. Retrieved from:

http://93.174.95.29/main/1194000/64bb25ec20662of 0366594375c40438a/Glenn\%20F.\%20Knoll\%20\%20Radiation\%20Detection\%20and\%20Measurement -Wiley\%20\%282010\%29.pdf

Retrieved on: Jun. 17, 2019

4. N. Tsoulfanidis, Measurement and detection of radiation, 1st ed., New York (NY), USA: McGraw-Hill, 1983.

Retrieved from:

https://trove.nla.gov.au/work/24937117?q\&sort=holdi $\underline{\text { ngs }+ \text { desc\& }=1574102120578 \& \text { versionId }=30083295 \mathrm{gg}}$ et

Retrieved on: Jan. 27, 2019

5. C. Dujardin et al., "Needs, Trends, and Advances in Inorganic Scintillators," IEEE Trans. Nucl. Sci., vol. 65, no. 8, pp. 1977 - 1997, Aug. 2018. DOI: $10.1109 /$ TNS.2018.2840160

6. A. Pushak et al., "Luminescent properties of BaCl2-Eu microcrystals embedded in a CsI matrix," Radiat. Meas., vol. 56, pp. 402 - 406, Sep. 2013.

DOI: 10.1016/j.radmeas.2013.01.071

7. D. M. Slaughter, C. R. Stuart, R. F. Klaass, D. B. Merrill, "Performance of Large Neutron Detectors Containing
Lithium-Gadolinium-Borate Scintillator," IEEE Trans. Nucl. Sci., vol. 63, no. 3, pp. 1650 - 1658, Jun. 2016. DOI: $10.1109 /$ TNS.2016.2561240

8. K. Yang, P. R. Menge, V. Ouspenski, "Li Co-Doped NaI:Tl (NaIL)-A Large Volume Neutron-Gamma Scintillator With Exceptional Pulse Shape Discrimination," IEEE Trans. Nucl. Sci., vol. 64, no. 8, pp. 2406 - 2413, Jun. 2017.

DOI: 10.1109/TNS.2017.2721398

9. P.W. Bridgman, "The Effect of Tension on the Transverse and Longitudinal Resistance of Metals," Proc. Amer. Acad. Arts Sci., vol. 6o, no. 8, pp. 423 449, Oct. 1925. DOI: $10.2307 / 25130064$

10. K. Kliemt, C. Krellner, "Crystal growth by Bridgman and Czochralski method of the ferromagnetic quantum critical material YbNi4P2," J. Cryst. Growth, vol. 449, pp. $129-133$, Sep. 2016.

DOI: 10.1016/j.jcrysgro.2016.05.042

11. V. B. Mikhailik, V. Kapustyanyk, V. Tsybulskyi, V. Rudyk, H. Kraus, "Luminescence and scintillation properties of CsI: A potential cryogenic scintillator," Phys. Status Solidi B, vol. 252, no. 4, pp. $804-810$, Jan. 2015.

DOI: $10.1002 / p s s b .201451464$

12. B. D. Milbrath, A. J. Peurrung, M. Bliss, W. J. Weber, "Radiation detector materials: An overview," J. Mater. Res., vol. 23, no. 10, pp. 2561 - 2581, Oct. 2008. DOI: 10.1557/JMR.2008.0319

13. M. Korzhik et al., "Detection of neutrons in a wide energy range with crystalline $\mathrm{Gd}_{3} \mathrm{Al}_{2} \mathrm{Ga}_{3} \mathrm{O}_{12}$, Lu2SiO5 and LaBr3 doped with Ce scintillators," Nucl. Inst. Meth. Phy. Res., vol. 931, pp. 88 - 91, Jul. 2019.

DOI: 10.1016/j.nima.2019.04.034 